\title{
The approximate analytical solution of the point source problem in 2D heterogeneous media
}

\author{
Zhifeng Liu ${ }^{1}$, Shengsheng Zhang ${ }^{1}$, Benoît Noetinger ${ }^{1}$, and Xiaohong Wang ${ }^{1}$ \\ ${ }^{1}$ University of Science and Technology of China
}

May 22, 2021

\begin{abstract}
The approximate analytical solution of the point source problem in $2 \mathrm{D}$ heterogeneous media is provided in this article. It is based on the analytical solution of $2 \mathrm{D}$ quasi-Laplace equation, which can be expressed as a combination of piecewise power series. Each power series is expanded at a singular point with a group of intrinsic non-integer power exponents, and then they are sewn together to obtain the solution for the whole region. To permit the power series to be used in the region containing point sources, the point source is approximately treated as a closed inner boundary, on which Neumann boundary condition is imposed. By this means, the approximate analytical solution of the point source problem, which is actually the Green's function for $2 \mathrm{D}$ Poisson equation, is obtained.
\end{abstract}

\section{Hosted file}

Article_submit_2.docx available at https://authorea.com/users/415115/articles/523029-theapproximate-analytical-solution-of-the-point-source-problem-in-2d-heterogeneous-media 